\title{
The Mode of Detection Is Not Associated with Quality of Life in Women with Breast Cancer
}

\author{
Bettina Braun ${ }^{a} \quad$ Marc-André Kurosinski $^{a} \quad$ Laura Khil $^{b} \quad$ Joke Tio ${ }^{c}$ \\ Barbara Krause-Bergmann ${ }^{d}$ Hans-Werner Hense ${ }^{a}$

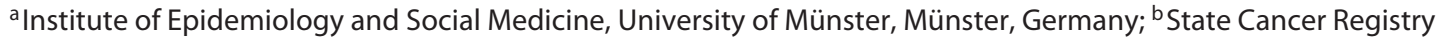 \\ of North Rhine-Westphalia, Bochum, Germany; ' Breast Care Center, Department of Gynecology and Obstetrics, \\ University Hospital Münster, Münster, Germany; ${ }^{\mathrm{d} D e p a r t m e n t}$ for Breast Diseases, St. Franziskus Hospital, \\ Münster, Germany
}

\section{Keywords}

Breast cancer screening - EORTC · Interval cancer - Mode of cancer detection - Quality of life

\begin{abstract}
Introduction: Apart from saving lives, mammography screening programs (MSP) are expected to reduce negative side effects of treatment by detecting cancer earlier, when it is more responsive to less aggressive treatment. This study compared quality of life (QoL) among women with breast cancers that were detected either by screening mammography, as interval cancers, or clinically among women not participating in the MSP. Methods: Retrospective study of firstever invasive breast cancers detected among MSP-eligible women aged 50-69 years between 2006 and 2012 in Münster, Germany. EORTC QLQ-C30 and -BR23 questionnaires were mailed to 1,399 cases still alive in 2015 (response rate 64.1\%). Results: Women's responses were obtained on average 6.1 years after diagnosis. Mean crude and age-adjusted scores for overall QoL, breast and body image (BBI), and five functional scales (FS) were comparable between groups of detection mode. Clearly lower adjusted means for most scores were observed in women with interval cancers, if time since diagnosis was less than 5 years. Cases younger than 60 years showed lower values for some FS, particularly among
\end{abstract}

interval and screen-detected cases. Discussion/Conclusion: In summary, cases with breast cancer showed health-related score values that were similar to the general population of the same age. There was also no indication that mode of detection markedly influenced these scores. However, after adjusting for tumor stage and other influential factors, screening participants appeared more susceptible to score declines after a diagnosis of cancer than non-participants.

(c) 2019 S. Karger AG, Basel

\section{Introduction}

As the number of long-term survivors has been rising consistently over recent years [1,2], research on survivorship of women with breast cancer has gained increasing attention [3]. Several studies assessed the quality of life (QoL) in long-term breast cancer survivors but their results were inconsistent [3-6]. Interestingly, the mode of breast cancer detection has rarely been associated with effects on QoL. The European Guidelines [7] emphasize that, apart from saving lives, breast cancer screening is also expected to reduce the negative side effects of treatment by detecting cancer when it is more responsive to less aggressive treatment. Therefore, we hypothesized that differences in the mode of breast cancer detection 
have an impact on women's QoL not only during the acute primary treatment phase but also in the medium and long term. Specifically, QoL in cancer cases detected by a mammography screening program (MSP) was expected to be better than in cancer cases detected clinically outside of the MSP. Furthermore, special emphasis was given to women with interval cancers, accounting for $25 \%$ of cases among MSP participants and commonly diagnosed in more advanced stages and with worse prognosis than screen-detected cancers $[8,9]$.

\section{Materials and Methods}

The German MSP started in late 2005. All German female residents in the age range of 50-69 years are eligible and receive invitations to attend the MSP every 2 years. This retrospective study included all MSP-eligible 1,534 female breast cancer cases in the age range of 50-69 years at diagnosis, who had newly detected first-ever breast cancer treated in one of the two certified breast care centers in Münster between 2006 and 2012. Date of diagnosis and first-line therapeutic management were extracted from the certified clinical tumor documentation system ONDIS [10] in the two breast care centers. Linkage with the population-based State Cancer Registry of North Rhine-Westphalia (LKR NRW) provided the mode of cancer detection and the vital status for each individual cancer case.

\section{Study Sample and Questionnaires}

Removing 135 deceased women (8.8\%) from the database, standardized questionnaires were mailed in 2015 to 1,399 women. Completed questionnaires were received from 897 women (64.1\%). We used only cases of invasive carcinoma $(n=735)$. Sociodemographic data, current predefined comorbidities, type of first-line breast cancer therapy, recent course of the disease, and current breast cancer therapy were obtained from self-reports and refer to the time of filling in the mailed questionnaires, considering that these factors are commonly associated with an influence on QoL. The EORTC QLQ-C30 Version 3.0 and parts of the QLQ-BR23 were used to assess QoL [11, 12]. Questions on sexual functioning originally contained in the EORTC QLQ-BR23 were deleted because we considered them as inappropriate in a mailed questionnaire. The functional scales (FS) of both questionnaires were scored from 0 to 100 with higher scores corresponding to better functioning. Score differences of more than 10 points were regarded as clinically relevant [13]. Symptom scores were also asked in the questionnaire but omitted from the present report based on the assumption that most of them (i.e., hair loss, nausea, and vomiting) are primarily relevant in the phase of primary treatment. Although studies show that some symptoms such as fatigue or lymphedema remain longer $[3,6]$.

\section{Statistical Methods}

Continuous variables were compared using the Student $t$ test and frequencies of categorized variables using a $\chi^{2}$ test. EORTC QLQ-C30 and -BR23 scores were analyzed based on the scoring manual $[11,14]$. Crude analyses compared the unadjusted mean values of overall QoL, breast and body image (BBI), and FS between detection groups, and also by age group and time since diagnosis. Factors associated with any of the scores $(p$ value $<0.01)$ were subsequently included in scale-specific linear regression models as single independent explanatory variables. The fully ad- justed models included all factors identified as influential in the preceding analytic steps; of note, however, factors resulting from screening participation and thus, reverse causality, i.e., breast-conserving therapy and self-reported chemotherapy, were not included. Analyses were carried out with the statistical software program SAS 9.4. The $p$ values reported here are products of multiple explorative tests and may not be interpreted as conclusive in terms of statistical significance. For more details, see the supplemental methods section (for all online suppl. material, see www. karger.com/doi/10.1159/000504662).

\section{Results}

Non-response analysis did not reveal major differences between respondents and non-responders with respect to age at survey and diagnosis, nodal stage, grading, and receptor status of tumors and therapeutic modalities (data not shown); however, tumors in non-responders were slightly more often in T2 stage ( 32.2 vs. $25.5 \%$ ).

The study sample consisted of women with screen-detected $(n=346)$, interval-detected $(n=88)$, and clinically detected $(n=301)$ breast cancers. The average age at survey was comparable across the three groups (Table 1). Time since diagnosis was greater (6.7 years) in breast cancer cases detected among non-participants than in screen-detected (5.6 years) and interval-detected cancer cases (5.7 years). For further differences between the groups, see Table 1.

Analyzing the association of potentially influential factors (Table 2), women younger than 60 years reported cognitive, emotional, and social functioning score values that were clearly lower than those in cases of older age. Of note, time since diagnosis was not distinctly associated with any of the QLQ scales. By contrast, depression, selfreported as comorbidity, exhibited a strong negative association with all FS, while diabetes mellitus, COPD, and osteoporosis related only to few specific scales. Breastconserving surgery (and closely related to it, radiotherapy) revealed a substantial positive relation to BBI, while past chemotherapy and current medical treatment showed a mild but consistently negative association with all scales. Generally, the QoL score was mostly related only to comorbidities but not by cancer therapy modalities, and fully adjusting for influential factors did not essentially alter the QLQ-C30 and -BR23 scale values.

Age-adjusted analyses of the QLQ-C30 and -BR23 scales produced similar mean score values for all three cancer detection modes. Likewise, adjusting additionally for all influential factors did not indicate any major differences between the detection modes (Fig. 1).

As age at survey and time since diagnosis differed between the detection mode groups, we applied a sensitivity analysis stratifying the fully adjusted models by age at survey and by time since diagnosis. Comparing scores among cases that were interviewed less than 5 years and 
Table 1. Characteristics of study participants with invasive breast cancer $(n=735)$, stratified by mode of detection

\begin{tabular}{|c|c|c|c|c|c|c|c|c|c|}
\hline & \multicolumn{2}{|c|}{$\begin{array}{l}\text { Screen-detected } \\
\text { cancer }\end{array}$} & \multicolumn{2}{|c|}{$\begin{array}{l}\text { Interval-detected } \\
\text { cancer }\end{array}$} & \multicolumn{2}{|c|}{$\begin{array}{l}\text { Clinically detected } \\
\text { cancer among } \\
\text { non-participants }\end{array}$} & \multicolumn{2}{|c|}{$\begin{array}{l}\text { Total (all } \\
\text { respondents) }\end{array}$} & \multirow{2}{*}{$\begin{array}{l}p \text { value for } \\
\text { differences between } \\
\text { categories of cancer } \\
\text { detection mode }\end{array}$} \\
\hline & $n=346$ & $47.1 \%$ & $n=88$ & $12.0 \%$ & $n=301$ & $41.0 \%$ & $n=735$ & $100 \%$ & \\
\hline \multicolumn{10}{|l|}{ Socioeconomic factors (mean $\pm S D$ ) } \\
\hline Age at survey, years & $65.3 \pm 6.6$ & & $63.8 \pm 5.6$ & & $64.8 \pm 6.3$ & & $64.9 \pm 6.4$ & & 0.012 \\
\hline Time since diagnosis & $5.6 \pm 1.8$ & & $5.7 \pm 1.8$ & & $6.7 \pm 2.1$ & & $6.1 \pm 2.0$ & & $<0.0001$ \\
\hline Education & & & & & & & & & $<0.001$ \\
\hline Lower than secondary school & 153 & $44.2 \%$ & 29 & 33.0 & 86 & 28.6 & 268 & 36.5 & \\
\hline At least secondary school & 191 & $55.2 \%$ & 56 & 63.6 & 212 & 70.4 & 459 & 62.4 & \\
\hline Missing & 2 & $0.6 \%$ & 3 & 3.4 & 3 & 1.0 & 8 & 1.1 & \\
\hline Partnership status & & & & & & & & & 0.845 \\
\hline Single or other & 95 & $27.5 \%$ & 22 & 25.0 & 77 & 25.6 & 194 & 26.4 & \\
\hline In a partnership & 249 & $72.0 \%$ & 64 & 72.7 & 222 & 73.8 & 535 & 72.8 & \\
\hline Missing & 2 & $0.6 \%$ & 2 & 2.3 & 2 & 0.7 & 6 & 0.8 & \\
\hline \multicolumn{10}{|l|}{ Comorbidities } \\
\hline Diabetes & & & & & & & & & 0.008 \\
\hline Yes & 34 & $9.8 \%$ & 0 & 0.0 & 31 & 10.3 & 65 & 8.8 & \\
\hline No & 305 & $88.2 \%$ & 86 & 97.7 & 267 & 88.7 & 658 & 89.5 & \\
\hline Missing & 7 & $2.0 \%$ & 2 & 2.3 & 3 & 1.0 & 12 & 1.6 & \\
\hline Depression & & & & & & & & & 0.340 \\
\hline Yes & 50 & $14.5 \%$ & 12 & 13.6 & 55 & 18.3 & 117 & 15.9 & \\
\hline No & 288 & $83.2 \%$ & 75 & 85.2 & 240 & 79.7 & 603 & 82.0 & \\
\hline Missing & 8 & $2.3 \%$ & 1 & 1.1 & 6 & 2.0 & 15 & 2.0 & \\
\hline Osteoporosis & & & & & & & & & 0.950 \\
\hline Yes & 54 & $15.6 \%$ & 13 & 14.8 & 47 & 15.6 & 114 & 15.5 & \\
\hline No & 277 & $80.1 \%$ & 74 & 84.1 & 250 & 83.1 & 601 & 81.8 & \\
\hline Missing & 15 & $4.3 \%$ & 1 & 1.1 & 4 & 1.3 & 20 & 2.7 & \\
\hline Chronic obstructive pulmonary disease & & & & & & & & & 0.014 \\
\hline Yes & 38 & $11.0 \%$ & 1 & 1.1 & 26 & 8.6 & 65 & 8.8 & \\
\hline No & 299 & $86.4 \%$ & 84 & 95.5 & 272 & 90.4 & 655 & 89.1 & \\
\hline Missing & 9 & $2.6 \%$ & 3 & 3.4 & 3 & 1.0 & 15 & 2.0 & \\
\hline \multicolumn{10}{|l|}{ Therapeutic modalities } \\
\hline Breast-conserving surgery & & & & & & & & & 0.033 \\
\hline Yes & 250 & 72.3 & 53 & 60.2 & 197 & 65.4 & 500 & 68.0 & \\
\hline No & 94 & 27.2 & 35 & 39.8 & 104 & 34.6 & 233 & 31.7 & \\
\hline Missing & 2 & 0.6 & 0 & 0.0 & 0 & 0.0 & 2 & 0.3 & \\
\hline Radiotherapy & & & & & & & & & 0.492 \\
\hline Yes & 283 & 81.8 & 71 & 80.7 & 235 & 78.1 & 589 & 80.1 & \\
\hline No & 63 & 18.2 & 17 & 19.3 & 66 & 21.9 & 146 & 19.9 & \\
\hline Hormone therapy & & & & & & & & & 0.622 \\
\hline Yes & 173 & 50.0 & 46 & 52.3 & 162 & 53.8 & 381 & 51.8 & \\
\hline No & 173 & 50.0 & 42 & 47.7 & 139 & 46.2 & 354 & 48.2 & \\
\hline Chemotherapy & & & & & & & & & $<0.0001$ \\
\hline Yes & 125 & 36.1 & 58 & 65.9 & 159 & 52.8 & 342 & 46.5 & \\
\hline No & 221 & 63.9 & 30 & 34.1 & 142 & 47.2 & 393 & 53.5 & \\
\hline \multicolumn{10}{|l|}{ Current medical treatment } \\
\hline Yes & 167 & 48.3 & 41 & 46.6 & 89 & 29.6 & 297 & 40.4 & $<0.0001$ \\
\hline No & 175 & 50.6 & 47 & 53.4 & 206 & 68.4 & 428 & 58.2 & \\
\hline Missing & 4 & 1.2 & 0 & 0.0 & 6 & 2.0 & 10 & 1.4 & \\
\hline
\end{tabular}

more than 5 years after diagnosis, there were no substantial differences between MSP participants with screendetected breast cancer and cancer cases among non-participants (Table 3a). However, interval-detected cases interviewed less than 5 years after diagnosis reported clinically relevant lower scores for BBI, cognitive, emotional, role and social functioning than interval-detected cases that had been diagnosed before more than 5 years. Moreover, these values were clearly lower than those of screen- and clinically detected cases (Table 3a). 


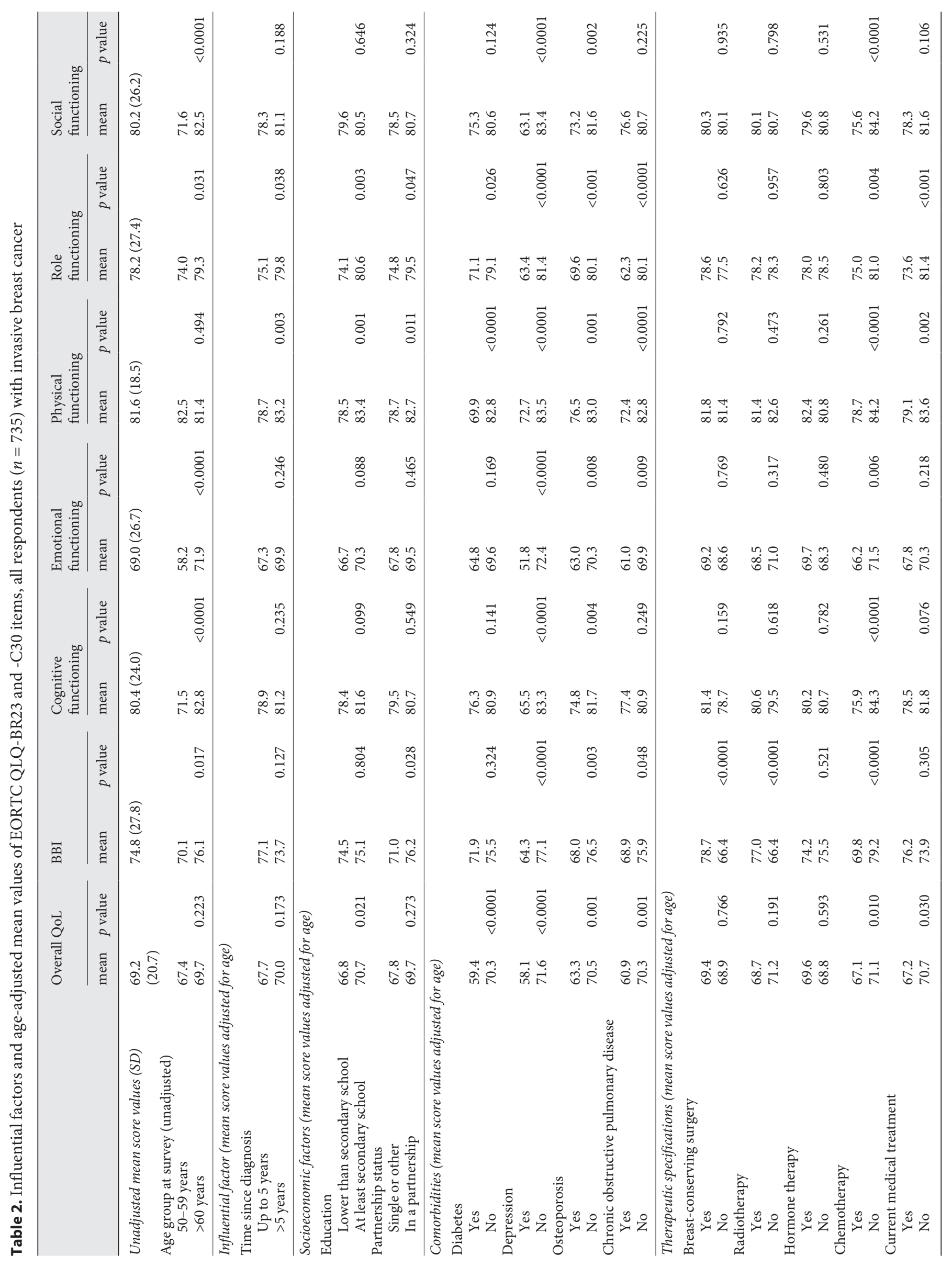




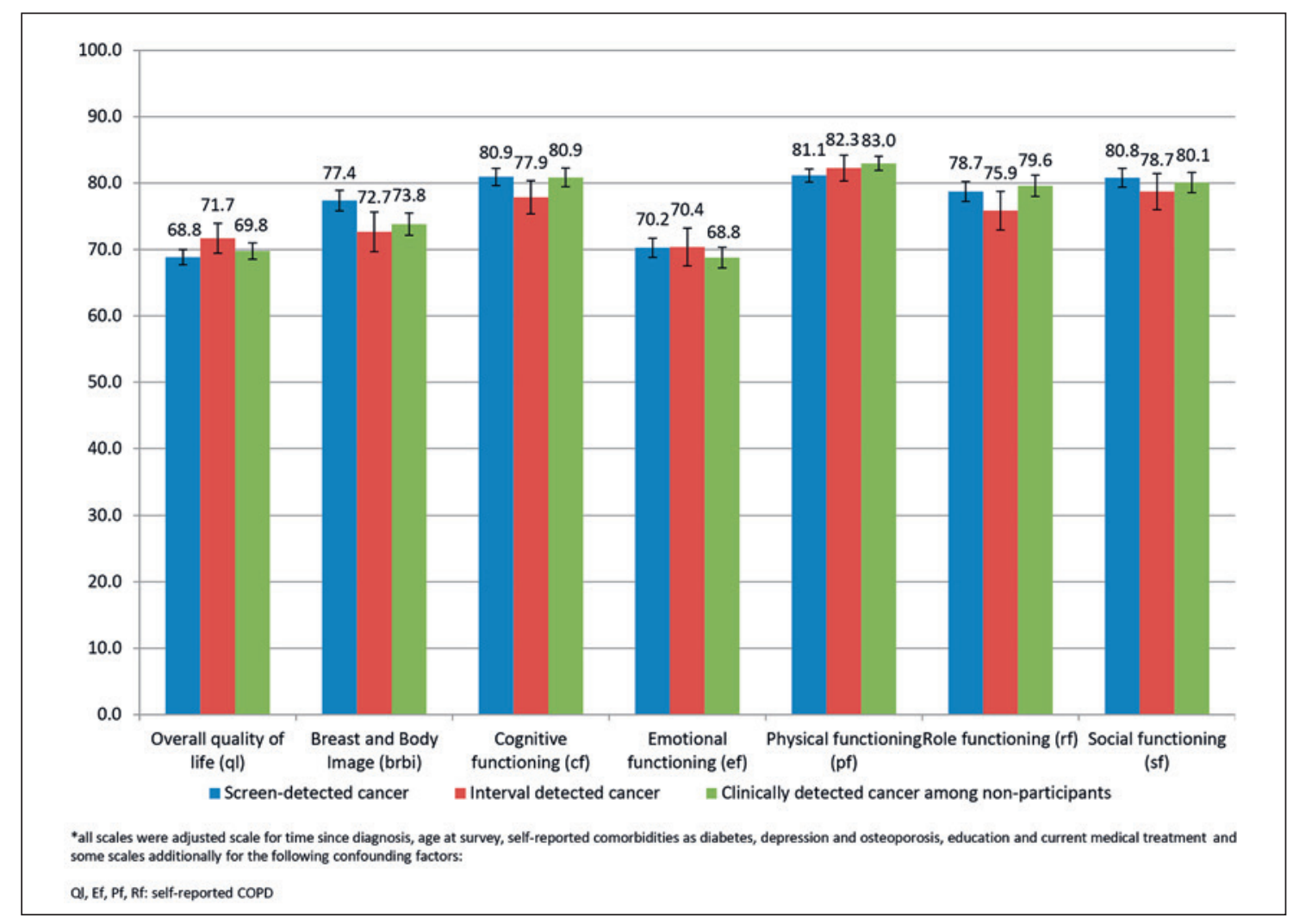

Fig. 1. Mean values and standard errors of the EORTC QLQ-BR23 and -C30 items, fully adjusted for significant factors (from Tables 1 and 2), by mode of detection.

Comparing the impact of detection modes by age group, younger women ( $50-59$ years) with screen-detected cancer reported markedly lower cognitive and emotional functioning, as well as moderately decreased role and social functioning, as compared to older women ( $>60$ years) (Table $3 b$ ). Similarly, low scores were also observed among younger interval cases as well as among younger non-participants with breast cancer, but these differences were less pronounced.

\section{Discussion/Conclusion}

This is, to our knowledge, the first study that investigated whether the detection mode of breast cancer is associated with global QoL and several functional parameters. We found no differences in age-adjusted scores between the three detection modes nor after adjustment for additional influential factors. Sensitivity analyses discovered a lowering score impact of age at and of time since diagnosis which differed between detection mode groups.

Differences in time since diagnosis between the groups may be due to the fact that the MSP was successively implemented from 2006 onwards and, at the beginning of the study period, mainly non-participants with breast cancer were treated in the two breast centers. This imbalance disappears over the years, but is still reflected in the longer overall time since diagnosis.

We reported before [15] that breast cancers detected by screening mammography were more often early stage cancers and that this was reflected by differences in primary and current cancer treatment. We therefore omitted influential factors such as breast-conserving therapy and self-reported chemotherapy as a consequence of screening participation from the fully adjusted model to avoid subtracting effects resulting from reverse causality. Notably, 'current medical treatment', that is essentially endocrine therapy, was not considered as a consequence of screening participation as the receptor status between MSP participants and non-participants was similar in our study population [15]. However, adjustment for the remaining influential factors did not materially alter the mean QoL and functional scores in the three groups of detection mode. A comparison with normative studies in German general populations $[16,17]$ and another study in German breast cancer patients [4] revealed mean scores for QoL, body image, and five FS that were close to those reported in these investigations. Scores that were similar to surveys of the general population $[14,15]$ seem to indicate that QoL and functioning assessed on average 


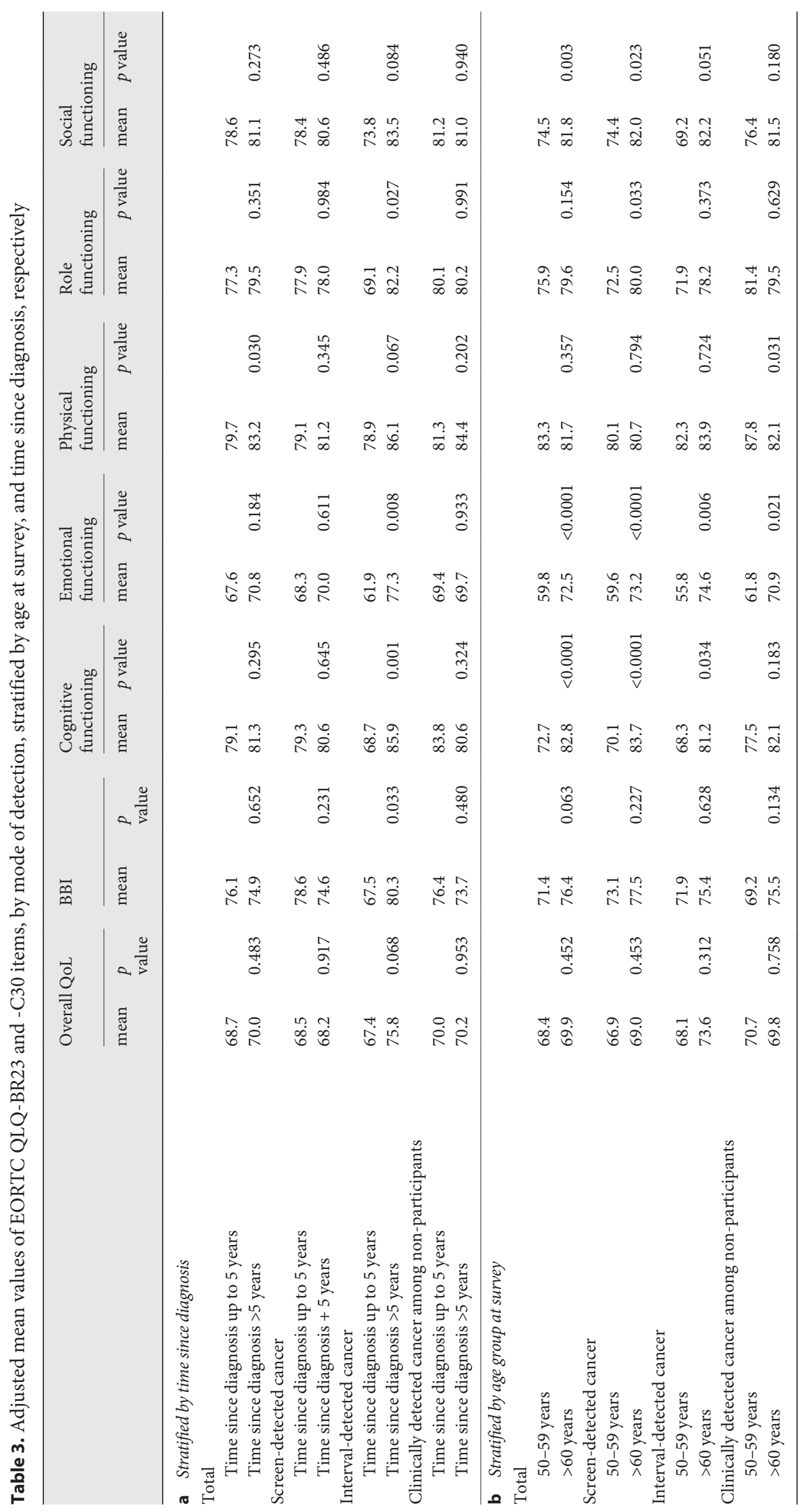


5-6 years after the diagnosis are, at least on a group level, no longer affected by the previous cancer diagnosis and treatment. Of note, there were persistent restrictions in some dimensions of the QoL of breast cancer survivors, but these differences were of rather low clinical significance $[3,18]$. Likewise, it is in particular conceivable that minor disparities between the three detection groups faded away over the years. This would be in line with studies on long-term cancer survivors $[2,3,19]$ showing that QoL depends strongly on how much time has passed since diagnosis. While primary surgical and medical interventions substantially affect QoL in the early months after diagnosis, QoL and functional scores seem to return to almost normal levels about 1 year after terminating cancer therapy [5].

The influence of time since diagnosis was rather modest in our study. Interestingly, the only pronounced disparity in score values was observed in women with interval cancers diagnosed before less than 5 years. This effect was apparently broad as the score decrements reached clinical relevance for most scales. Interestingly, these score differences were fully adjusted, including factors such as advanced tumor stage. One may hypothesize that other subjective factors may have exerted specific influences here. Thus, MSP participants with an interval breast cancer - despite an inconspicuous screening mammography only a few months before - possibly react with a feeling of deception and exceptional concern that affect their QoL adversely for a longer time. This finding points to a particular vulnerability of interval cancer cases.

Earlier studies have also shown that older women rated their mental and physical function better than younger women $[3,20]$. Stratification by age in this study revealed that lower, clinically relevant scores were specifically present among MSP participants, both in screen- and interval-detected cases, which were younger than 60 years. Thus, younger women, voluntarily opting for a screening mammography, appear more susceptible to disappointment and anxiety evoked by the cancer diagnosis than cases of the same age who had decided not to follow the screening invitations.

A recent study looked into factors that were related to the uptake of the German MSP [21, 22]. In a questionnaire survey, MSP participants tended to be more concerned with acute threatening aspects of breast cancer, they also overestimated the benefits of mammography screening, and assumed that mammography screening offered the best protection against breast cancer [21]. Therefore, MSP participants might feel a greater degree of deception when they are diagnosed with breast cancer - more so if diagnosed in the interval.

There are several aspects of this study that need to be considered when interpreting the results. The response rate of $64.1 \%$ is comparatively high and the non-response analysis revealed no major response bias. The instruments used in the questionnaires are standardized and have been used before in clinical and epidemiological studies, thus suggesting a valid and reliable database. Nevertheless, self-reports may be prone to bias and misclassification [23]. However, we cannot conceive how social desirability or inaccurate recall should have distorted our comparison because there is no obvious reason why participants and non-participants of the MSP should tend to differ in the way they report their QoL. As noted above, symptom scores were omitted from the present report based on the assumption that they reflect mostly the early phase of cancer treatment which was not covered in this study population. Similarly, we deleted the questions on sexual functioning from the BR23 questionnaires as we doubted the validity of self-reported sexual function in a mailed questionnaire. More importantly, we do not have data on QoL before breast cancer diagnosis as it would allow assessing changes subsequent to the cancer diagnosis and enable control of self-selection bias of MSP participants. We noted, however, that the potentially influential factors were mostly balanced between the groups of detection mode. A further limitation may be seen in the fact that our study database was derived only from patients of two breast care centers in one German city and that it may have a limited external applicability.

In summary, there was no indication that mode of detection markedly influenced health-related QoL and FS. Breast cancer cases among MSP participants, whether detected at screening or in the interval, were more adversely affected when they were younger and, more specifically for interval cancer cases, if the cancer diagnosis was more recent. The results seem to indicate that, after adjusting for tumor stage and other influential factors, screening participants are more susceptible to a decline in QoL after a diagnosis of cancer than non-participants.

\section{Acknowledgement}

Dr. Andrea Fuhs and Dr. Oliver Heidinger were involved in discussing the initial study concepts. Dr. Ron Pritzkuleit, Prof. Dr. Annika Waldmann, and Laura Buschmann, MSc, commented on early versions of the manuscript.

\section{Statement of Ethics}

The research was conducted in accordance with the Declaration of Helsinki.

The study protocol was approved by the medical ethical commission of the medical council of Westphalia-Lippe and the medical faculty of the Westphalian Wilhelms University Münster (reference 2015-139-f-S). Informed consent was obtained from all participants included in the study. 


\section{Disclosure Statement}

The authors have no conflicts of interest to declare.

\section{Funding Sources}

The study was conducted with the institute's own financial resources.

\section{References}

1 Mols F, Vingerhoets AJ, Coebergh JW, van de Poll-Franse LV. Quality of life among longterm breast cancer survivors: a systematic review. Eur J Cancer. 2005 Nov;41(17):2613-9.

2 Mehnert A, Koch U. Psychological comorbidity and health-related quality of life and its association with awareness, utilization, and need for psychosocial support in a cancer register-based sample of long-term breast cancer survivors. J Psychosom Res. 2008 Apr;64(4): 383-91.

3 Koch L, Jansen L, Herrmann A, Stegmaier C, Holleczek B, Singer S, et al. Quality of life in long-term breast cancer survivors - a 10 -year longitudinal population-based study. Acta Oncol. 2013 Aug;52(6):1119-28.

4 Waldmann A, Pritzkuleit R, Raspe H, Katalinic A. The OVIS study: health related quality of life measured by the EORTC QLQ-C30 and -BR23 in German female patients with breast cancer from Schleswig-Holstein. Qual Life Res. 2007 Jun;16(5):767-76.

5 Schmidt ME, Wiskemann J, Steindorf K. Quality of life, problems, and needs of disease-free breast cancer survivors 5 years after diagnosis. Qual Life Res. 2018 Aug;27(8): 2077-86.

6 Arndt V, Stegmaier C, Ziegler H, Brenner H. A population-based study of the impact of specific symptoms on quality of life in women with breast cancer 1 year after diagnosis. Cancer. 2006 Nov;107(10):2496-503.

7 Perry N, Broeders M, de Wolf C, Törnberg S, Holland R, von Karsa L. European guidelines for quality assurance in breast cancer screening and diagnosis. Fourth edition - summary document. Ann Oncol. 2008 Apr;19(4):61422 .

\section{Author Contributions}

All authors were involved in the development and implementation of the study. All authors read and commented drafts of the manuscript and agreed on the final version. Dr. Kurosinski was specifically responsible for the management of the study database. Dr. Khil was responsible for linking data from the state cancer registry. Dr. Tio and Dr. Krause-Bergmann were in charge of the breast cancer centers recruiting the study participants. Mrs. Braun was responsible for analyzing the data and writing the first draft of the manuscript. Prof. Dr. Hense is the principal study investigator.
8 Heidinger O, Batzler WU, Krieg V, Weigel S, Biesheuvel C, Heindel W, et al. The incidence of interval cancers in the German mammography screening program: results from the population-based cancer registry in North Rhine-Westphalia. Dtsch Arztebl Int. 2012 Nov;109(46):781-7.

9 Houssami N, Hunter K. The epidemiology, radiology and biological characteristics of interval breast cancers in population mammography screening. NPJ Breast Cancer. 2017 Apr;3(1):12

10 ONDIS - Ihr Steuerungsinstrument in der onkologischen Qualitätssicherung Dortmund: Kassenärztliche Vereinigung Westfalen-Lippe; 2019 [cited 2019 Nov 5]. Available from: https://www.kvwl.de/arzt/ehealth/ it/ondis.htm

11 Fayers P, Aaronson NK, Bjorda K, Groenvold M, Curran D, Bottomley D. EORTC QLQC30 Scoring Manual. 3rd ed. Brussels: European Organisation for Research and Treatment of Cancer; 2001.

12 Scott NW, Fayers PM, Aaronson NK, Bottomley A, de Graeff A, Groenvold $\mathrm{M}$, et al. EORTC QLQ-C30 Reference Values Manual. 2nd ed. Brussels, Belgium: EORTC Quality of Life Group; 2008.

13 Osoba D, Rodrigues G, Myles J, Zee B, Pater J. Interpreting the significance of changes in health-related quality-of-life scores. J Clin Oncol. 1998 Jan;16(1):139-44.

14 Sprangers MA, Groenvold M, Arraras JI, Franklin J, te Velde A, Muller M, et al. The European Organization for Research and Treatment of Cancer breast cancer-specific quality-of-life questionnaire module: first results from a three-country field study. J Clin Oncol. 1996 Oct;14(10):2756-68.

15 Braun B, Khil L, Tio J, Krause-Bergmann B, Fuhs A, Heidinger $\mathrm{O}$, et al. Differences in Breast Cancer Characteristics by Mammography Screening Participation or Non-Participation. Dtsch Arztebl Int. 2018 Aug;115(3132):520-7.
16 Waldmann A, Schubert D, Katalinic A. Normative data of the EORTC QLQ-C30 for the German population: a population-based survey. PLoS One. 2013 Sep;8(9):e74149.

17 Schwarz R, Hinz A. Reference data for the quality of life questionnaire EORTC QLQ$\mathrm{C} 30$ in the general German population. Eur J Cancer. 2001 Jul;37(11):1345-51.

18 Doege D, Thong MS, Koch-Gallenkamp L, Bertram H, Eberle A, Holleczek B, et al. Health-related quality of life in long-term disease-free breast cancer survivors versus female population controls in Germany. Breast Cancer Res Treat. 2019 Jun;175(2):499-510.

19 Schoormans D, Czene K, Hall P, Brandberg Y. The impact of co-morbidity on health-related quality of life in breast cancer survivors and controls. Acta Oncol. 2015 May;54(5):72734.

$20 \mathrm{Wu}$ HS, Harden JK. Symptom burden and quality of life in survivorship: a review of the literature. Cancer Nurs. 2015 Jan-Feb; 38(1):E29-54.

21 Albert US, Fischbeck S, Schulte H, Klusendick M, Diener J. Wissenschaftlicher Bericht: Inanspruchnahme des qualitätsgesicherten Mammographie-Screenings - Follow-Up Studie 2012 [Scientific Report: Use of Quality Assured Mammography Screening - FollowUp Study 2012]. 2012.

22 Albert US, Kalder M, Schulte H, Klusendick M, Diener J, Schulz-Zehden B, et al. [The population-based mammography screening programme in Germany: uptake and first experiences of women in 10 federal states]. Gesundheitswesen. 2012 Feb;74(2):61-70. German

23 Choi BC, Pak AW. A catalog of biases in questionnaires. Prev Chronic Dis. 2005 Jan; 2(1):A13 